\title{
Explanatory Server Protection Problem for Automated Wireless System
}

\author{
Singh Poja Ramesh ${ }^{1}$, Santhosh Kumar Singh ${ }^{2}$ \\ ${ }^{1}$ Research Scholar, AMET University, Chennai \\ ${ }^{2}$ Assistant Professor,Tagore College of Science \& Commerce, Mumbai
}

\begin{tabular}{l} 
Article Info \\
\hline Article history: \\
Received Oct 19, 2017 \\
Revised Dec 24, 2017 \\
Accepted Jan 9, 2018 \\
\hline
\end{tabular}

Keywords:

Automated

Security Break and Security

Server

\begin{abstract}
Many sorts of servers exists which incorporates both openly available servers and inside servers, for example, mail servers, web servers, application servers, assemble and test servers and so forth., that store numerous private and delicate data. Giving security to such servers has turned out to be one of the center necessities in this day and age as they are under danger of assault. The information put away on the server can go from hierarchical data, for example, classified client related records, extend subtle elements, company's product source code to private media data, individual information, national security related data, patients database, address papers of the focused exams and so forth. On the off chance that such delicate information gets into wrong hands, it can be abused. Along these lines the business and the notoriety of the association would beat stake. It can likewise posture risk to countries. The fundamental reasons of security break in servers is because of the utilization of versatile stockpiling gadgets, for example, pen drives, hard circles, and so on., which can be potential transporters of malware and Trojans. At the point when tainted by such infection, these compact stockpiling gadgets can bring about serious harm. Physically keeping up and securing servers would be extremely troublesome and can likewise prompt tremendous authoritative overhead. Henceforth the requirement for computerized security system to distinguish, keep and shield the servers from the aggressors. Security strategies assume a critical part in keeping the trade off of system security which would influence the server security.
\end{abstract}

Copyright () 2018 Institute of Advanced Engineering and Science. All rights reserved.

\section{Corresponding Author:}

Singh Poja Ramesh, Research Scholar, AMET University, Chennai.

\section{INTRODUCTION}

The snappy improvement of PC systems has given incredible comfort and also presented security dangers in the figure and capacity condition. Since we are constantly associated with Internet we at no time in the future can be guaranteed of the information being secure. A summary of attack methods and confidentiality protection measures for fully automated remote analysis systems is illustrated in [1].

Different sorts of information are moved and put away in freely available systems [2], for example, servers or distributed storage, which might be wrongfully captured, changed or harmed by assailants bringing about the altering or loss of information.

Servers which are under hazard and very helpless are real section focuses for noxious clients. [3] Diverse sorts of security components have been created to shield the servers from different sorts of assaults. Security instrument implements all the required security strategies [4]. On the off chance that anybody figures out how to discover any escape clause in the server which can be abused, it would prompt bargain of server and information [5]. Once the helplessness has been found the influenced frameworks must be fixed 
quickly else the harm could be tremendous. A portable mobile-based complete blood count (CBC) analysis framework with the aid of microscope is proposed, and the smartphone camera is mounted to the viewing port of the light microscope by adding a smartphone support. Then, the number of corresponding cells are counted using topological structural analysis, and the cells in clumped region is estimated using Hough Circle Transform (HCT) procedure. After that, the analysis results are saved in the database, and shown in the user interface of the smartphone application [6].

\section{PROPOSED METHOD}

This paper proposes a structure that would facilitate the work of an overseer. It concentrates on planning a mechanized device which would play out a review of the servers and check in the event that it is agreeable to all the recommended security arrangements. As there are numerous stages whereupon the servers run, the apparatus is intended to adjust to heterogeneous condition.

The security arrangements which were tried incorporated the accompanying:-

Checking if the Windows server is running the endorsed cutting-edge against malware arrangement.

Checking if the endorsed antivirus shield is found in the taskbar for a framework running Windows.

List the rendition of the Anti-Malware running on the frameworks for Linux and Windows working framework. Media Access Delay and Throughput Analysis of Voice Codec with Silence Suppression on Wireless Ad Hoc Network is described in [7]. Check if any individual removable media introduce for both Linux and Windows Server, if so list their names and timestamp of the gadget addition. K-strange points clustering algorithm. In Computational Intelligence in Data Mining is determined in [8].

List all the security patches connected to the Windows working framework.

List the introduced forms of programming running on the Windows System and Check if the occasion logs have been empowered or crippled. A survey of big data analytics in healthcare and government is described in [9]. The security device would help the overseer to check the assemble and test server for security consistence and ensuring that it sticks to all the characterized security arrangements.

\section{RESULT AND DISCUSSION}

Container technology solves a number of problems for any software business including improved cost efficiency, data isolation, improved data portability, and versatility.

\section{PROBLEMS}

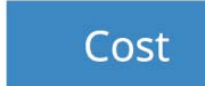

\section{Security}

\section{Data}

Versatility

\section{SOLUTIONS}

Stratus 5 automates deployment, delivery, and monetization of Docker containers improving efficiency, and reducing costs.

Most secure Docker platform. High level protection against hackers, data breaches, and customer data exposure.

Stratus5 automates backups, encryption, data migration, and geo-distribution.

Stratus 5 is cloud agnostic, and can easily migrate data between any cloud provider without data loss.

Figure 1. Container Technology 


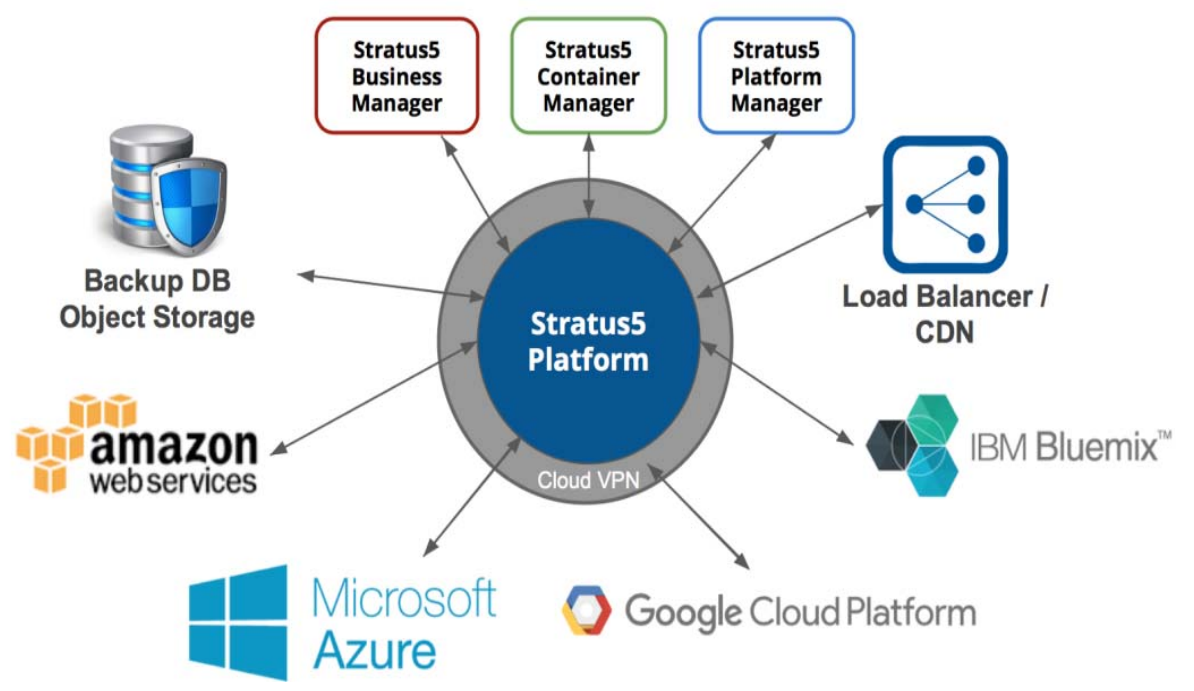

Figure 2. Stratus5

Stratus 5 automates most of the processes required to manage Docker containers and cloud servers, as well as including additional business service automation for software as a service companies.

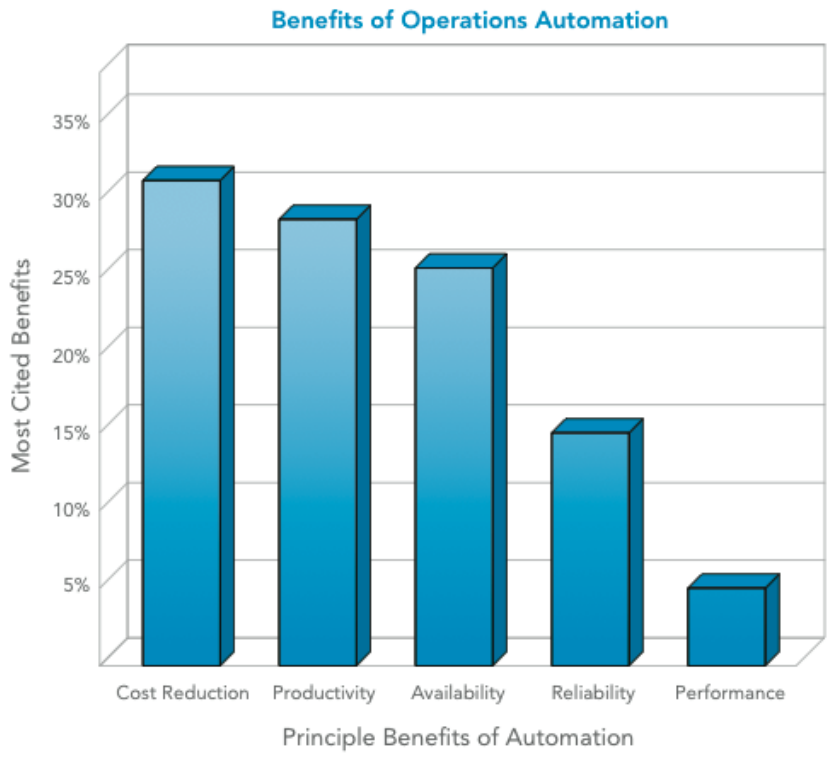

Figure 3. Benefits of Automation

The primary benefits of operations automation cited most often were cost reduction, productivity, availability, reliability, and performance. Every business faces global pressure to increase their profitability. One approach is to reduce costs. But, reducing the capabilities of the computer center negatively impacts the entire company.

\section{CONCLUSION}

Associations must utilize standard security standards and must guarantee the checking of the logs so that any follows left by an interloper can be followed and the security dangers can be found before they make any real harm the organization. The dangers must be broke down and their example must be recorded to 
counteract future assaults. Information ruptures and interruption assaults can't be ceased out and out yet we should make important strides in ensuring the servers in a heterogeneous domain. Thus security must be given the most astounding need as a procedure in every one of the associations and must be rehearsed in a taught way. The point of the work was to outline a compelling strategy consistence apparatus which would check if the servers were in consistence with all the recommended arrangements. This would enable the executive to settle the escape

\section{REFERENCES}

[1] O'Keefe C. M., "A summary of attack methods and confidentiality protection measures for fully automated remote analysis systems," International Statistical Review, vol/issue: 81(3), pp. 426-455, 2013.

[2] Sabin D., U.S. Washington D. U.S, “Patent and Trademark Office,” Patent No. 6,981,207, 2005.

[3] Bouwman H., et al., "Opportunities and problems with automated data collection via smartphones," Mobile media \& communication, vol/issue: 1(1), pp. 63-68, 2013.

[4] Zaitsev O. Washington, DC: U.S, "Patent and Trademark Office," U.S. Patent No. 8,776,241, 2014.

[5] Olsen T., "Eu Data Protection Regulation and Automatic Processing of Information on The Internet," 2002.

[6] C. Y. Kit, et al., "Mobile based Automated Complete Blood Count (Auto-CBC) Analysis System from Blood Smeared Image," Indonesian Journal of Electrical Engineering and Computer Science, vol/issue: 7(6), pp. 3020 3029, 2017.

[7] Shah R. D. and Singh S. K., "Media Access Delay and Throughput Analysis of Voice Codec with Silence Suppression on Wireless Ad Hoc Network," Procedia Computer Science, vol. 79, pp. 940-947, 2016.

[8] Johnson T. and Singh S. K., "K-strange points clustering algorithm," in Computational Intelligence in Data Mining, vol. 1, pp. 415-425, 2015.

[9] Archenaa J. and Anita E. M., "A survey of big data analytics in healthcare and government," Procedia Computer Science, vol. 50, pp. 408-413, 2015. 